\title{
Wheeler, Tiomno e a Física Brasileira
}

\author{
(Wheeler, Tiomno, and Brazilian Physics) \\ José Maria Filardo Bassalo* \\ Departamento de Física da UFPA, 66075-900, Belém, Pará, Brasil \\ Olival Freire Junior ${ }^{\dagger}$ \\ Instituto de Física da UFBA, 40210-340, Salvador, BA, Brasil \\ Recebido em 01 Outubro, 2003. Aceito em 31 de outubro, 2003.
}

\begin{abstract}
Este artigo examina as relações do físico norte-americano John Archibald Wheeler com o físico brasileiro Jayme Tiomno. A imagem de Tiomno como um dos físicos teóricos mais brilhantes de sua geração emerge amplificada; mas fica claro, também, que os prejuízos causados pelo regime militar (1964-1985) ao desenvolvimento da ciência brasileira foram ainda maiores do que os que usualmente se reconhece.
\end{abstract}

This paper analyzes the relationships between the American physicist, John Archibald Wheeler, and the Brazilian physicist Jayme Tiomno. It enlarges Tiomno's image as one of the most gifted theoretical physicist of his generation and it reveals that the losses of the Brazilian science, due to the military government (1964-1985), were more meaningful than what one usually admits.

\section{Introdução}

I always think of Tiomno as one of the most unappreciated of physicists. His work on muon decay and capture in 1947-1949 was pathbreaking and would still merit recognition by some suitable award. J. A. Wheeler, 1998.

Neste artigo, examinaremos as relações do físico norteamericano John Archibald Wheeler com o físico brasileiro Jayme Tiomno, e com a Física brasileira, tendo como pano de fundo uma apresentação das principais idéias e conceitos físicos formulados por Wheeler ao longo de sua carreira de pesquisador. Tomamos como ponto de partida a autobiografia de Wheeler, a qual nos fornece um panorama de sua trajetória intelectual,[1] marcada por uma permanente inconformidade com as idéias já estabelecidas na ciência, além de um interessante quadro da Física e de seu contexto, ao longo de um vasto período entre 1930 e 1990. O livro revela também um fragmento da história da física brasileira, com um expressivo depoimento a propósito da carreira científica de Tiomno. A consulta aos arquivos pessoais de Wheeler permite ampliar significativamente a informação sobre as relações entre Wheeler e a Física brasileira, e sugere uma interessante reflexão tanto sobre a pujança intelectual quanto sobre as vicissitudes características da nossa ciência.[2] A imagem de Tiomno como um dos físicos teóricos mais brilhantes de sua geração emerge amplificada desse estudo; mas fica claro, também, que os prejuízos causados pelo regime militar ao desenvolvimento da ciência brasileira foram ainda maiores do que os que usualmente reconhecemos.

\section{Wheeler: uma vida entre partículas, campos e informação}

Wheeler nasceu em Jacksonville, Flórida, no dia 9 de julho de 1911. Ao entrar na Universidade Johns Hopkins, em 1927, seu interesse inicial foi em Engenharia em virtude de suas leituras em livros sobre Ciência e Tecnologia. Contudo, ao folhear livros de Engenharia na Biblioteca dessa Universidade deparou-se com exemplares da Zeitschrift für Physik que tratavam da recente Mecânica Quântica e sua aplicação ao comportamento de elétrons atômicos. As discussões que teve com seus professores norte-americanos, o de Química H. M. Smallwood, e os de Física John C. Hubbard e seu assistente R. Bowling Brown, sobre o que estudara daquela Mecânica fizeram-lhe decidir ser um físico. Assim, passou para o Departamento de Física da Universidade Johns Hopkins e, em 1933, recebeu o título de Doutor em Física, tendo o físico austríaco Karl Ferdinand Herzfeld como orientador. Entre 1933 e 1935, trabalhou com o físico norte-americano Gregory Breit na Universidade de Nova York e com o físico dinamarquês Niels Henrik David Bohr [Prêmio Nobel de Física (PNF), 1922] em Copenhague, na Dinamarca. Em 1935, assumiu o cargo de Professor Assistente na Universidade de Carolina do Norte ao aceitar o convite do físico norte-americano Arthur E. Ruark. Transferiu-se para a Universidade de Princeton, em 1938, na qual ficou até 1976, depois de ser Joseph Henry Professor nos últimos dez anos.

\footnotetext{
*bassalo@amazon.com.br

†freirejr@ufba.br
} 
Neste ano, mudou-se para Austin, no Texas, aceitando a proposta do físico indiano-norte-americano Ennackel Chandy George Sudarshan para ser professor na Universidade do Texas, em Austin. Nesta, assumiu em 1979 a Cadeira de Física Ashbel Smith e, em 1981, tornou-se Blumberg Professor. Aposentou-se em 1986. Registre-se que, em 1968, ele ganhou o Prêmio Fermi, da Comissão de Energia Atômica Americana, em 1982, foi agraciado com a Niels Bohr International Gold Medal, e em 1997, recebeu o Prêmio Wolf.

Em sua recente autobiografia, Wheeler divide sua carreira científica em três fases, assim por ele denominadas: Everything Is Particles ("Tudo É Partículas"); Everything Is Fields ("Tudo É Campos"); Everything Is Information ("Tudo É Informação"). Na primeira delas, que vai desde o começo de sua carreira científica até 1952, ele desenvolveu a tese de que todas as partículas até então conhecidas poderiam ser constituídas a partir da junção do elétron e de sua antipartícula, o pósitron, junção essa que seria mediada pelo fóton, segundo preconizava a Teoria Quântica da Radiação, formulada pelo físico inglês Paul Adrien Maurice Dirac (PNF, 1933), em 1927. Esse bi-elétron, como ele o denominou, que também fora concebido, de maneira independente, por Ruark e pelo físico russo Lev Davidovich Landau (PNF, 1962), foi pela primeira vez observado pelo físico norte-americano Martin Deutsch, no Massachusetts Institute of Technology (MIT), em 1951, e é hoje conhecido como positrônio.[3] Para evitar que o seu bi-elétron fosse instável, Wheeler foi levado a admitir uma ação à distância, em um mundo sem fótons e formado somente de partículas. Essas idéias foram desenvolvidas com a colaboração do então jovem e talentoso físico norte-americano Richard Phillips Feynman (PNF, 1965),[4] na primeira metade da década de 1940, e foram publicadas na Physical Review 59, p. 683, em 1941, e na Reviews of Modern Physics (RMP) 17, p. 157, em 1945, e 21, p. 425, em 1949. Nesses trabalhos, a Eletrodinâmica é formalizada, por intermédio de um Princípio de Mínima Ação, em termos de partículas agindo a distância sem a necessidade do campo eletromagnético (fóton) intermediante.[5]

\section{Wheeler e a Física Brasileira}

Foi nessa primeira fase que se iniciou a relação de Wheeler com a Física brasileira. No artigo da RMP de 1949, escrito por Wheeler e Feynman, há uma citação dos artigos dos físicos brasileiros Mário Schenberg e José Leite Lopes [Physical Review 67, p. 122 (1945)] e de Schenberg [Summa Brasiliensis Mathematicae 1, números 5 e 6 (1946); Physical Review 74, p. 738 (1948)], nos quais eram analisados, basicamente, a teoria do elétron puntiforme e o problema das divergências na Eletrodinâmica Quântica.

Ainda nessa primeira fase da carreira científica de Wheeler,[6] na segunda metade da década de 1940, ele teve o segundo contato com a Física Brasileira. Vejamos como isso aconteceu. Em 1947, as experiências realizadas na Universidade de Bristol, na Inglaterra, pelos físicos, os ingleses Sir Cecil Frank Powell (PNF, 1950) e Hugh Muiread, o italiano Guiseppe Paolo Stanislao Occhialini, e o brasileiro César Mansuetto Giulio Lattes indicavam que os raios cósmicos eram constituídos de dois tipos de partículas: os mésons primários (hoje, píons) e os mésons secundários (hoje, múons). Imediatamente Wheeler interessou-se pelos múons, principalmente pelo seu decaimento e pela sua captura por núcleos atômicos. Por outro lado, Tiomno assistira no Brasil, ainda em 1947, a uma conferência que Lattes proferira sobre aquela famosa descoberta da qual participara. Como Wheeler, Tiomno logo se interessou pelos múons. Nessa ocasião, veio-lhe a idéia de que os múons também poderiam sofrer uma força do tipo-Fermi. Registre-se que, em 1934, o físico italiano Enrico Fermi (PNF, 1938) havia proposto que o decaimento beta (transformação do nêutron em próton, com a emissão de elétron e neutrino) era devido a uma nova força na Natureza, a força (interação) fraca.

Com a bolsa de estudos Buenos Aires Convention, concedida pelo United States Office of Education, dos Estados Unidos da América, Tiomno foi para a Universidade de Princeton, em 1948, realizar estudos de pós-graduação com Wheeler. Ao buscar Princeton para concluir sua formação em Física Teórica, Tiomno seguia uma trilha aberta por Schenberg, que ali estivera em 1940, com uma bolsa da Fundação Guggenheim, e Leite Lopes, que ali se doutorara sob a orientação do físico austro-suíço Wolfgang Pauli Junior (PNF, 1945). Wheeler era então um dos mais prestigiados físicos teóricos norte-americanos.[7] Conforme depoimento da física brasileira Elisa Frota-Pessoa, mulher de Tiomno, Wheeler costuma afirmar que os três estudantes com os quais trabalhou mais arduamente foram Feynman, Tiomno e o físico norte-americano Robert Eugene Marshak.[8] Inicialmente, começaram a trabalhar em Relatividade Geral. Contudo, como ambos já haviam pensado no decaimento dos múons, passaram então a analisar esse tipo de decaimento. Assim, juntos, desenvolveram a tese de atribuir spin $1 / 2$ ao múon, inicialmente apresentada no Centennial Meeting of the American Association for Advancement of Science, realizado em Washington, DC, no dia 15 de setembro de 1948, e desenvolvida nos artigos publicados no Reviews of Modern Physics 21, pgs. 144 e 153, em 1949. Segundo Wheeler, essa tese foi sugerida por Tiomno, sob a forma de um triângulo, cujos vértices eram constituídos dos núcleons [próton $(p)$ e nêutron $(n)$ ], do elétron $\left(e^{-}\right)$e de seu companheiro neutrino (hoje, $\nu_{e}$ ), do múon $(\mu)$ e de seu provável companheiro, a partícula leve e neutra $\nu_{0}$ (hoje, $\left.\nu_{\mu}\right)$. Segundo o esquema apresentado naqueles artigos, as interações entre dois desses pares de partículas seriam do tipo-Fermi.

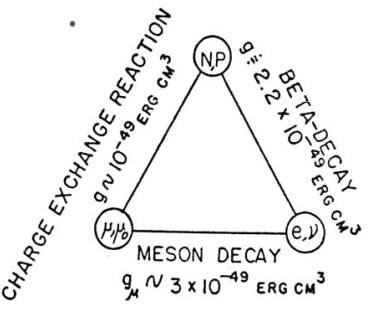

Tiomno \& Wheeler, RMP, 21(1), 1949

Nesta oportunidade, é interessante fazermos algumas observações sobre a fortuna do reconhecimento da 
contribuição de Tiomno e sobre a atitude de Wheeler em face desse reconhecimento. É bem aceito na literatura da Física e da História da Física que a hipótese de que qualquer dos três pares de partículas $[(p, n) ;(e, \nu) ;(\mu, \nu)]$ interagiriam igualmente consigo ou com quaisquer dos outros pares, um processo de captura e decaimento, foi um caso de descobertas independentes e simultâneas. Os físicos norteamericanos Val Logsdon Fitch (PNF, 1980) e Jonathan L. Roser,[9] por exemplo, atribuem essa hipótese, denominada de "weak universality", aos seguintes físicos: o ítalo-russo Bruno M. Pontecorvo; o sueco Oskar Benjamin Klein; o italiano Giampietro Puppi; os sino-norte-americanos Tsung Dao Lee (PNF, 1957) e Chen Ning Yang (PNF, 1957) e o norte-americano Marshal N. Rosenbluth; e Tiomno e Wheeler. Desse ponto de vista, a contribuição de Tiomno teve pleno reconhecimento na Física do Século XX. Contudo, apenas Tiomno, como assinalado por Wheeler,[10] expressou esta idéia na forma de um diagrama - o referido triângulo publicado no artigo conjunto de Tiomno e Wheeler - capaz de expressar sinteticamente o conhecimento físico e matemático implícito na proposta. Em particular, Puppi, em 1948, apresentou idéias semelhantes a essas de WheelerTiomno no Nuovo Cimento 5, p. 587, sem, contudo, fazer qualquer diagrama. Apesar disso, o reconhecimento dessa contribuição de Tiomno tem sido incerto. O problema remonta ao início da década de 1950, quando o físico francês Louis Michel, em artigo de revisão, publicou o desenho do triângulo, mas sem atribuir-lhe autoria,[11] foi agravado na década seguinte, quando passou a ser denominado de triângulo Puppi-Tiomno,[12] e atingiu seu ápice quando Marshak, no início da década de 1980, falando em um evento que resultou em uma obra clássica da História da Física de Partículas, denominou-o apenas de triângulo Puppi.[13] Nessa altura, em 1984, Tiomno reagiu, participando, instado por Wheeler, de conferência dedicada aos 50 anos da interação fraca, realizada em Racine, EUA.[14] Retornaremos a esse episódio adiante, mais aqui deve ser ressaltado que tanto a sua participação nessa conferência quanto as cartas trocadas em seguida com o próprio Marshak colocaram um freio a esta nada sutil mudança na atribuição de prioridades.[15] A presença de espírito de Tiomno ficou evidente quando intitulou uma seção de sua apresentação como "the case of the mutating triangle". Em obra ulterior, Marshak não mais atribuiu o triângulo a Puppi, mas o apresentou como o "famoso triângulo das interações fracas" sem atribuí-lo a qualquer autor.[16] Em face da incerteza desse reconhecimento é que se pode compreender a ênfase posta por Wheeler em sua autobiografia, publicando um desenho com o triângulo, e afirmando que ele deveria se chamar apenas triângulo Tiomno pelas razões expostas acima. As palavras de Wheeler, pelo seu significado, merecem ser transcritas:

"I always thought that this triangle should be called the 'Tiomno triangle'. He got there first. But a few months after our paper appeared, Giampietro Puppi published similar ideas in an Italian journal. He, too, saw the great simplicity of a common interaction among nucleons, electrons, muons, and neutrinos. As luck would have it, the Tiomno triangle is now known to everyone as the 'Puppi triangle', even though
Puppi did not include a diagram in his paper”. [17]

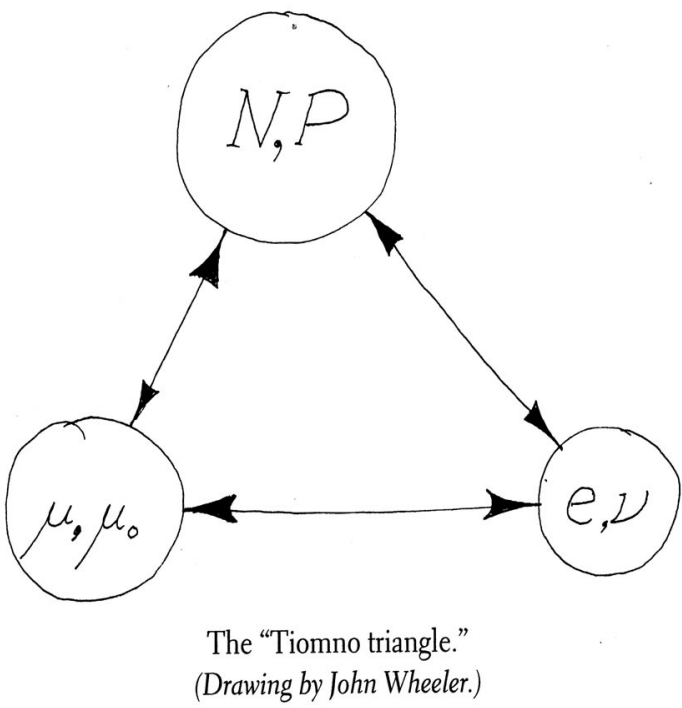

Provavelmente motivado pela tomada de posição de Wheeler, Tiomno, recentemente, abordou o problema em discurso por ocasião da concessão do "Marcel Grossman Institutional Award" ao Centro Brasileiro de Pesquisas Físicas (CBPF), no Rio de Janeiro, em julho de 2003. A originalidade Tiomniana desse triângulo está interessantemente relacionada com um outro triângulo, desta vez político, da vida desse cientista brasileiro - Rio de Janeiro-Brasília-São Paulo - pelo físico brasileiro Sérgio Joffily, por ocasião da homenagem prestada aos 80 anos de Tiomno no XXIEncontro Nacional de Física de Partículas e Campos, realizada em São Lourenço, Minas Gerais, no dia 25 de outubro de 2000. A razão desse "triângulo político de Tiomno" o leitor entenderá mais adiante.

Observe-se, ainda, que a universalidade da interação tipo-Fermi foi pela primeira vez formalizada em artigo de Tiomno e Yang, publicado na Physical Review 79, p. 495, em 1950. Aliás, foi esse artigo que cunhou o termo "interação universal" de Fermi, utilizado por longo período nos artigos que tratam do que hoje se conhece apenas como interação fraca. Por fim, destaquemos que Tiomno defendeu sua Tese de Doutoramento, intitulada "Teorias do Neutrino e a Dupla Desintegração Beta", na Universidade de Princeton, em 1950, tendo como orientador o físico húngaro-norteamericano Eugene Paul Wigner (PNF, 1963), pois Wheeler viajara para Paris, em junho de 1949, com uma bolsa da Fundação Guggenheim, para realizar pesquisas em Paris e em Copenhague.

Antes de passarmos para a fase Everything Is Fields, o que ocorreu em 1952, conforme frisamos antes, Wheeler desenvolveu um trabalho pioneiro sobre estrutura nuclear, tendo ainda como tema a tese Everything Is Particles. Com efeito, desde que trabalhou com Bohr no modelo da "gota líquida", em 1939, segundo já relatamos, Wheeler continuou discutindo com esse físico sobre a forma do núcleo atômico. Assim, com a colaboração do físico norte-americano David Lawrence Hill, ele formulou um modelo nuclear em que os núcleons apresentavam um movimento independente no 
interior do núcleo, o qual denominou de "modelo coletivo do núcleo", e que serviria para explicar a fissão nuclear. Dias antes de viajar para Paris, Wheeler enviou para Bohr um rascunho desse trabalho, o qual apresentava a autoria compartilhada pelos três físicos. Encontrando-se em Paris, Wheeler viajou para Copenhague, em setembro de 1949, para debater com Bohr o rascunho que lhe enviara. Nessa ocasião, discutiram como ajustar os modelos "gota líquida" e "coletivo" para explicar os recentes resultados sobre a deformação do núcleo, traduzido pelo cálculo do momento de quadrupolo nuclear realizado, ainda em 1949, pelos físicos norte-americanos Charles Hard Townes (PNF, 1964), Henry Michael Foley e W. Law.

Em dezembro de 1949, Wheeler continuou examinando com Bohr, agora por intermédio de cartas, como conciliar os dois modelos nucleares. No ano seguinte, Wheeler decidiu não publicar a idéia que tivera sobre o modelo "coletivo", já que pretendia ampliar o rascunho do trabalho que iniciara com Bohr e Hill. Contudo, nesse mesmo ano de 1950, ele teve uma grande decepção ao receber um trabalho preliminar ("preprint") (publicado ainda em 1950 na Physical Review 79, p. 432) do físico norteamericano Leo James Rainwater o qual mostrava que o núcleo atômico deveria apresentar uma forma não-esférica como conseqüência de um efeito coletivo. Este físico fora levado a esse modelo ao perceber que o cálculo do momento de quadrupolo das terras raras não era explicado pelo então vigente Modelo Nuclear em Camadas, esboçado pela física alemã Maria Goeppert Mayer (PNF, 1963), em 1948, e completado por ela própria, e independentemente, pelos físicos alemães Johannes Hans Daniel (PNF, 1963) e Otto Haxel, e pelo geoquímico austríaco Hans Eduard Suess, em 1949. Destaque-se que o hoje famoso Modelo Coletivo Nuclear foi desenvolvido pelo físico dinamarquês Aage Niels Bohr, filho de Bohr, em 1951, e ainda por Aage e pelo físico norte-americano-dinamarquês Ben Roy Mottelson, em 1953. Quando o PNF de 1975 foi atribuído a Rainwater, Aage e Mottelson pela formulação daquele modelo, Wheeler fez o seguinte comentário para si próprio, e agora reproduzido no livro Geons, Black Holes and Quantum Foam, citado acima: "Quando alguém descobre alguma coisa significante, é melhor publicá-la imediatamente e não esperar para incorporá-la em algum grande esquema. Esperar reunir todas as peças pode ser certo para o filósofo, mas não é sensato para um físico".

\section{Everything is Fields}

Agora, passemos à segunda fase, Everything Is Fields, da vida científica de Wheeler. Em janeiro de 1952, ele começou a estudar os clássicos trabalhos realizados, em 1939, pelos físicos, os norte-americanos Julius Robert Oppenheimer e Hartland Snyder, e o russo George Michael Volkoff sobre o colapso estelar. Segundo esses físicos, quando todas as fontes termonucleares de energia são exauridas de uma estrela suficientemente pesada, então a contração gravitacional continuará indefinidamente até seu colapso total. Esta "singularidade" - um ponto geométrico de densidade infinita - é uma conseqüência real da Teoria da Relatividade, ainda segundo aqueles físicos; ela já havia sido tratada pelo astrônomo alemão Karl Schwarzschild, em 1916, razão pela qual ficou conhecida como singularidade de Schwarzschild.

Quando Wheeler iniciou o ano acadêmico de 19531954, em Princeton, no qual ensinaria a Teoria da Relatividade,[18] começou a pensar na Terceira Lei de Newton, a da ação e reação, e seu papel na famosa confirmação da Relatividade Geral de Einstein, qual seja, a do encurvamento da luz ao passar pelo Sol, observado no eclipse de 1919, e previsto por essa Teoria. Assim, para Wheeler, se a luz é influenciada pela gravidade, então a gravidade seria influenciada pela luz, conforme aquela lei Newtoniana. Desse modo, pensou então que a luz não só é afetada pela gravidade, mas, também, ela própria pode criar gravidade. Aliás, isso não era novidade, observou Wheeler, uma vez que a Relatividade Restrita Einsteiniana, indicava que, como toda energia é convertível em massa, a energia poderia ser então uma fonte de gravidade. Desse modo, veio-lhe a idéia de criar uma entidade hipotética, o geon (g de "gravidade", e de "eletromagnetismo", e on da palavra raiz de "partícula" - elétron, próton, nêutron, méson, píon etc.). Tal entidade significava o seguinte: a luz circulando em torno de um centro e mantida por sua própria gravidade. Ou seja, ela representava um campo gravitacional feito inteiramente de campo eletromagnético, isto é, uma entidade "massiva sem massa". Wheeler continuou a trabalhar nessa idéia e, em 1954, esboçou seu primeiro texto sobre o geon - um toro ("rosca") de luz do tamanho do Sol com a massa equivalente a milhões de sóis - e o enviou a Einstein. Em conversa telefônica, Einstein disse-lhe que já havia pensado numa entidade desse tipo, energia comprimida, porém em tamanho muito menor. No entanto, a descartara por ser "não-natural". Apesar de suas equações relativistas permitirem uma solução desse tipo, esta não seria estável, concluiu Einstein naquela conversa telefônica.

Embora esse texto que Wheeler enviara a Einstein fosse clássico, pois baseou-se na Relatividade Geral Einsteiniana, já havia nele um traço quântico, pois nele especulou a possibilidade de haver um fenômeno quântico que pudesse mudar a natureza do geon e, em consequiência, ele passaria a radiar energia, como ocorre com o par elétron-pósitron. O trabalho sobre geons de Wheeler, publicado em 1955 (Physical Review 97, p. 511), tratou de geons esféricos e em forma de toros ("roscas"), constituídos de luz e, também, de neutrinos. Mais tarde, Wheeler chegou a desenvolver um geon puro, constituído apenas de energia gravitacional, em torno do qual ondas gravitacionais (que decorrem das equações de Einstein) de fantástica intensidade giravam, criando bastante gravidade para mantê-las juntas. Uma das coisas que fascinava Wheeler na sua proposta do geon é que este, diferentemente das partículas até então conhecidas (elétrons, prótons e mésons), apresentava uma estrutura interna e não era apenas uma entidade infinitesimal como estas últimas partículas representam. E mais, em grandes distâncias, o geon se comportava como uma fonte pontual de gravidade. Para Wheeler, essa entidade poderia ser um estado de transição entre a onda gravitacional e o colapso gravitacional, previsto por Oppenheimer, em 1939, e denominado de buraco negro ("black hole") por Wheeler, em 1967, conforme veremos 
mais adiante.

A idéia de "massa sem massa" não foi a única apresentada por Wheeler no artigo que escreveu sobre o geon, já que nele havia, também, a idéia de "carga sem carga", que decorria da seguinte questão por ele apresentada: se a Teoria Quântica controla o campo elétrico, o campo magnético e o campo neutrino, não poderia ela também controlar o campo gravitacional, e o próprio espaço-tempo gerador deste último? A resposta a esta questão foi dada por Wheeler por intermédio dos conceitos de buraco de minhoca ("wormhole") e de espuma quântica ("quantum foam"), em trabalhos subseqüentes, e, com isso, gerou sua fase Everything Is Fields.[19] A discussão do papel da geometria na gravitação levou Wheeler à formulação da "Dinâmica da Geometria" - a Geometrodinâmica -, em artigo publicado na Reviews of Modern Physics 33, p. 63, em 1961, o qual tem como tese fundamental o seguinte: "A massa maneja o espaço ensinando-o a curvar-se, e o espaço maneja a massa ensinando-a como mover-se". Aquela expressão foi cunhada por ele em analogia com a Eletrodinâmica, teoria esta que mostra como o campo eletromagnético é criado e propagado. Um estudo completo dessa nova teoria foi apresentado por Wheeler no livro intitulado Geometrodynamics, publicado pela Academic Press, em 1962.

Na seqüência de seu estudo sobre a Teoria da Relatividade Geral e sua relação com a Teoria Quântica, Wheeler voltou a analisar o problema do colapso gravitacional estelar, que havia examinado em 1952 quando se preparava para ensinar a Relatividade na Universidade de Princeton, conforme relatamos anteriormente. Dessa análise, surgiu a discussão sobre o conceito de buraco negro ("black hole"). Em agosto de 1967, a astrônoma irlandesa Susan Jocelyn Bell Burnell, então estudante do astrônomo inglês Antony Hewish (PNF, 1974), encontrou objetos celestes na nebulosa de Caranguejo que emitiam vibrações regulares de ondas de rádio, com o período aproximado de 1,337 segundos, e que, jocosamente, chamou-os de LGM ("Little Green Men"). No outono desse mesmo ano, o físico italiano Vittorio Canuto, então chefe administrativo do Goddard Institute for Space Sudies, da NASA, sediado em New York, convidou Wheeler para fazer uma conferência objetivando uma possível interpretação dessa descoberta. Em um certo instante de sua exposição, na qual argumentava sobre a possibilidade de o centro de tais objetos ser um "objeto colapsado completamente pela gravidade", alguém da platéia sugeriu um nome mais compacto: "How about black hole"? Como procurara desesperadamente por um nome compacto para descrever aquela situação física, Wheeler aceitou-o e passou a adotá-lo oficialmente, no dia 29 de dezembro de 1967 , na conferência realizada na Sociedade Sigma X-Phi Beta Kappa, sediada ainda em New York. Na literatura científica, o nome "black hole" apareceu nos artigos que Wheeler publicou no American Scholar 37, p. 248 e no American Scientist 56, p. 1, ambos em 1968.

É oportuno destacar que o colapso gravitacional e o "black hole" foram objeto de estudo por parte de Wheeler e colaboradores. Com efeito, em 1965, Wheeler e os físicos, os norte-americanos B. Kent Harrison e Thorne, e o japonês Wakano trataram do colapso gravitacional no livro intitulado
Gravitation Theory and Gravitational Colapse, publicado pela University of Chicago Press. Por sua vez, Wheeler e o físico italiano Remo Ruffini discutiram o "black hole" em artigo publicado no Bulletin of the American Physical Society 15, p. 76, em 1970. Nesse artigo, há a conjectura de que esse objeto é extremamente simples visto de fora, já que ele só pode influenciar os objetos ao seu redor por intermédio de sua massa, carga e spin, e nada mais. Essa conjectura foi demonstrada, na década de 1970, nos diversos artigos dos físicos, os ingleses Stephen William Hawking e Brandon Carter, e o germano-canadense Werner Israel, e ficou conhecida como o famoso Teorema: O "buraco negro" não tem cabelo.[20]

\section{As Vicissitudes da Física Brasileira durante o Regime Militar}

Enquanto Wheeler desenvolvia os trabalhos em Gravitação descritos anteriormente e que correspondem à fase Everything Is Fields, seus amigos físicos brasileiros, Schenberg, Tiomno e Leite Lopes, eram perseguidos pelo golpe militar brasileiro de 31 de março de 1964, já que tiveram dificuldades administrativas para exercer o magistério em suas respectivas instituições de ensino: Schenberg, na Universidade de São Paulo (USP), Tiomno e Leite Lopes, no Centro Brasileiro de Pesquisas Físicas (CBPF) e na Faculdade Nacional de Filosofia (FNFi), ambos no Rio de Janeiro. Schenberg, por exemplo, foi preso sete dias depois do golpe e permaneceu confinado, no Departamento de Ordem Política e Social (DOPS) de São Paulo, durante dois meses até conseguir um "habeas corpus" que o permitiu voltar às suas atividades na USP.[21] No CBPF, o então diretor, Octacílio Cunha, tentando adaptar-se aos novos tempos do regime militar, procurava criar toda sorte de empecilhos às atividades de físicos como Leite Lopes e Tiomno, os quais passaram a buscar outras instituições para dar continuidade a suas atividades científicas. Leite Lopes foi trabalhar na Faculté de Sciences, em Orsay, na França, a convite do físico francês Maurice Lévy, e Tiomno ficou no Brasil aguardando se concretizar o convite que o físico brasileiro Roberto Aureliano Salmeron lhe fizera para dirigir o Instituto Central de Física, na Universidade de Brasília (UnB). Salmeron dirigia o Instituto Central de Ciências dessa Universidade.

Cartas trocadas entre Leite Lopes e Wheeler, respectivamente, em 15 de dezembro de 1964 e 4 de janeiro de 1965, indicam a preocupação de Wheeler com a situação da física brasileira, em particular com a de seus amigos físicos, pois tinha grande respeito pela contribuição que eles deram à Física. Um exemplo desse respeito foi manifestado por Wheeler, ainda em 1957, quando Tiomno foi indicado para receber o primeiro Prêmio Moinho Santista de Ciências Exatas, em 1957. Em 9 de abril de 1957, o físico austro-brasileiro Guido Beck consultou Wheeler sobre a concessão dessa alta honraria nacional a Tiomno. Sobre essa consulta, Wheeler respondeu, em 24 de maio de 1957, o seguinte: "It appears true, that in the whole hemisphere of the earth south of the equator, Tiomno is the most distinguished scientist concerned with the theory of ele- 
mentary particle transformations and causality". Registre-se que, além dos importantes trabalhos que Tiomno havia realizado sobre a interação fraca, com Wheeler e com Yang, no final da década de 1940 e referidos anteriormente, ele fizera, com o físico brasileiro Walter Schutzer, o trabalho intitulado On the Connection of the Derivative Matrices with Causality, publicado na Physical Review 83, p. 249, em 1951, e que havia sido citado pelo físico ítalo-norte-americano Nicola N. Khuri, em 1957 (Physical Review 107, p. 1148), como sendo uma "pedra fundamental" na Física das Partículas Elementares. Esse trabalho resolveu, pela primeira vez, a questão de como introduzir uma condição de causalidade no formalismo matemático e verificar suas implicações sobre a matriz $S$. Em vista disso, esse artigo foi considerado como um dos precursores da Teoria da Dispersão pelo físico norteamericano Murphy Goldberger, em 1969, por ocasião da comemoração dos quinze anos dessa Teoria. Essa referência encontra-se no livro Subnuclear Phenomena, Volume 1, editado por A. Zichichi, e publicado pela Academic Press, em 1970.

Tiomno foi para Brasília, em março de 1965 e, a seu convite, um dos autores do presente artigo (JMFB), iniciou, ainda nesse mês, sua carreira de físico. Infelizmente, a pressão dos militares brasileiros sobre a UnB resultou na destruição dessa instituição superior de ensino, em outubro de 1965, um processo cujos detalhes foram descritos por Salmeron em seu livro A Universidade Interrompida: Brasília 1964-1965, Editora UnB, 1999. Em vista disso, Tiomno voltou para o Rio de Janeiro. Como não havia mais ambiente científico para ficar no Rio de Janeiro, por causa das perseguições políticas que estavam ocorrendo no CBPF e na FNFi, Tiomno começou a pensar na possibilidade de trabalhar na USP.

Preocupado com a situação de seu amigo Tiomno, Wheeler convidou-o, por duas vezes, em 13 de novembro e 18 de dezembro de 1967, para ser Professor Visitante na Universidade de Princeton. Contudo, como acabara de conquistar a Cadeira de Física Teórica e Superior, do Departamento de Física da Faculdade de Filosofia Ciências e Letras, da USP (FFCL/USP), em novembro de 1967, Tiomno declinou desse honroso convite, em carta escrita a Wheeler, em 8 de janeiro de 1968 .

Na FFCL/USP, Tiomno reuniu vários professores (JMFB, entre eles) objetivando criar um novo grupo de pesquisas nessa universidade estadual. Este grupo está na origem do atual Departamento de Física Matemática, o qual foi implantado pelo físico brasileiro Hersh Moysés Nussenzveig.[22] Mais uma vez, a intolerância do regime militar brasileiro, agora de posse de um instrumento ditatorial, o ato institucional número 5 (AI-5), de 13 de dezembro de 1968, interrompeu essa nova etapa da carreira científica de Tiomno ao aposentá-lo, juntamente com outros professores brasileiros (sua mulher Elisa Frota Pessoa, Leite Lopes e Schenberg, dentre outros), em abril de 1969, em decorrência do ato complementar número 75 . Wheeler tomou conhecimento desse ato arbitrário ao receber uma carta do físico brasileiro Roland Köberle, datada de 28 de abril de 1969.

De posse dessa informação, Wheeler começou a escre- ver cartas para várias pessoas a fim de ver o que poderia fazer em prol de seus amigos. Assim, no dia 5 de maio de 1969, Wheeler contactou com o Conselho da American Physical Society para verificar a possibilidade de esta associação saber do embaixador brasileiro Mário Gibson Barbosa a razão daquele arbítrio e, se possível, revertêlo. Em 14 de maio de 1969, o físico norte-americano W. W. Havens Junior, secretário executivo daquele Conselho, dirigiu-se ao embaixador Barbosa solicitando informações sobre as circunstâncias em que ocorreram as aposentadorias de Tiomno e Leite Lopes, sem, presumivelmente ("allegedly"), qualquer oportunidade de defesa por parte desses distintos físicos ("distinguished physicists"). A mesma preocupação foi manifestada por Wheeler em cartas que escreveu, em 16 e 19 de maio de 1969, respectivamente, para Salmeron, que se encontrava em Paris, e para o físico brasileiro José Goldemberg, que ensinava na Escola Politécnica, em São Paulo. Ainda em maio, no dia 21, Leite Lopes escreveu uma carta a Wheeler comunicando-lhe que, em virtude das aposentadorias de diversos físicos brasileiros e do clima de insegurança acadêmica que a ditadura militar tinha implantado no Brasil, não haveria mais a XI Escola Latino-Americana de Física, que aconteceria no Rio de Janeiro, de 2 a 30 de julho de 1969, sob sua Coordenação, e, portanto, estava cancelado o convite que ele fizera, em 17 de setembro de 1968, para Wheeler falar sobre o presente e o futuro da Teoria da Relatividade Geral.

Registre-se que manifestações de outras personalidades internacionais sobre a aposentadoria compulsória dos professores brasileiros, em particular de Tiomno e Leite Lopes, foram endereçadas ao presidente-militar Artur da Costa e Silva. Dentre tais manifestações, destacam-se o telegrama de 31 de maio de 1969 enviado pelo norte-americano Lincoln Gordon, então presidente da Universidade Johns Hopkins, e o de Yang, no dia 5 de junho de 1969. A manifestação de Lincoln Gordon era particularmente relevante porque, como embaixador dos Estados Unidos no Brasil, entre 1961 e 1966, teve ativa participação no apoio norte-americano ao golpe militar.[23] Além disso, Costa e Silva recebeu telegramas de protesto contra tais aposentadorias contendo a assinatura de cerca de 600 físicos de várias nacionalidades.

Observamos, incidentalmente, que a extensão da solidariedade norte-americana às vítimas do regime militar brasileiro, especialmente a partir de 1968, ainda está por receber a devida apreciação por parte dos nossos historiadores. A mudança de posição de Lincoln Gordon, por exemplo, recebeu destaque no New York Times, em $1^{\circ}$ de junho de 1969, com a manchete Ex-u.s. aide joins protest to Brazil - Gordon and Others Experts Assails Faculty Purges". A matéria prossegue, afirmando "a former high State Department official once closely identified with United States support for the military-dominated government in Brazil has joined American professors in a written protest against recent faculty purges in Brazilian universities. The document was cabled to Arthur da Costa e Silva, President of Brazil today". O documento continha mais de trezentas assinaturas, e um documento similar, com 283 assinaturas, também foi enviado ao governo brasileiro.[24] Entender a mudança na posição de Lincoln Gordon nos remeteria à distinção esta- 
belecida pelo jornalista brasileiro Elio Gaspari, ao denominar seus dois livros recentes de A Ditadura Envergonhada (Companhia das Letras, 2002), para se referir ao período até fins de 1968, e A Ditadura Escancarada (Companhia das Letras, 2002), para o período posterior até o início do Governo Geisel.

Apesar dessas manifestações, a situação dos professores aposentados permaneceu a mesma, o que ensejou Wheeler a enviar uma carta a Tiomno, no dia 24 de junho de 1969 , convidando-o para trabalhar na Universidade de Princeton, no ano acadêmico de 1969-1970. De Trieste, na Itália, onde se encontrava realizando pesquisas no International Center for Theoretical Physics, a convite de seu grande amigo, o físico paquistanês Abdus Salam (PNF, 1979) (criador, juntamente com Tiomno e outros físicos internacionais desse Centro),[25] Tiomno escreveu, no dia 8 de agosto de 1969, uma carta para Wheeler dizendo-lhe que pretendia discutir pessoalmente esse convite, em sua volta ao Brasil, via Estados Unidos. Contudo, esse encontro não aconteceu, já que Wheeler lhe prevenira, em carta de 21 de agosto, que não estaria em Princeton no período proposto por Tiomno, mas recomendou a Goldberger que o recebesse.

$\mathrm{Na}$ expectativa de que a tempestade-militar que desabara sobre o Brasil era passageira, Tiomno voltou ao Brasil declinando daquele convite. No Brasil, um convite formulado por seus antigos discípulos, colaboradores e admiradores, os professores brasileiros Jorge André Swieca (já falecido), Nicim Zagury, Antônio Luciano Leite Videira e Erasmo Madureira Ferreira, para trabalhar no Departamento de Física da Pontifícia Universidade Católica do Rio de Janeiro (PUC/RJ), esbarrou em obstáculos políticos derivados da proibição, imposta pelo regime militar, de trabalhar em qualquer instituição de ensino brasileiro.[26] Tiomno continuou produzindo trabalhos científicos indicando sua residência na rua Alexandre Ferreira, no Jardim Botânico, no Rio de Janeiro, como o local para o qual os interessados deveriam se dirigir para solicitar os reprints desses trabalhos.

Preocupado com o rumo que a Física Brasileira tomaria com o afastamento de seus melhores físicos, Wheeler escreveu, no dia 22 de julho de 1969, uma carta a Nussenzveig, que se encontrava em Rochester, sobre a proposta do Deão Robert Fuller, do Trinity College, em Hartford, Connecticut, relativa à possibilidade de criação de um Instituto de Pesquisa na América do Sul, com sede no Brasil. Essa atitude de Wheeler, bem como a sua preocupação com seus amigos aposentados, fizeram com que Leite Lopes, então professor visitante na Carnegie-Mellon University, em Pittsburg, Pennsylvania, para o ano acadêmico de 19691970, escrevesse, em 21 de outubro de 1969, uma carta para Wheeler agradecendo a solidariedade que prestara aos físicos brasileiros que foram punidos pela sandice da ditadura militar. A solidariedade de Wheeler com os físicos brasileiros não foi restrita àqueles com quem tinha relações pessoais próximas. Assim é que, em 12 de dezembro de 1970, informado por Nussenzveig e por Thorne da prisão, em São Paulo, do casal de físicos brasileiros Ernest e Amélia Hamburger, Wheeler enviou um telegrama ao presidente Emilio Médici, solicitando que fossem observados os direitos humanos e legais do casal, e expressando sua preocupação com a informação de que ambos estavam incomunicáveis.[27]

O recrudescimento da ditadura militar, em conseqüência da edição do AI-5 e de seus atos complementares, fez com que Tiomno finalmente decidisse sair do Brasil. Assim, em 18 de setembro de 1970, Tiomno recebeu uma carta do físico Freeman Dyson para ser Professor Visitante na Universidade de Princeton e no Instituto de Estudos Avançados, também situado na cidade de Princeton, no ano letivo de 1970-1971. No dia 24 de setembro, Tiomno responde a Dyson e a Wheeler que aceitava o honroso convite para trabalhar naquelas Instituições, nas quais ficou cerca de um ano e meio, entre 1971 e 1972 . A estada em Princeton foi bastante produtiva para Tiomno, levando à conclusão de 11 trabalhos científicos.[28] No entanto, a saudade do Brasil e de seus amigos foi tão grande que Tiomno e sua mulher Elisa decidiram voltar ao país. A carta de Tiomno a Wheeler, em 27 de junho de 1972, evidencia tanto o apreço mútuo entre eles quanto a importância dessa estada para a recuperação moral de Tiomno, depois das vicissitudes políticas sofridas no Brasil.[29] Como veremos adiante, os prejuízos causados por tais vicissitudes ainda não tinham terminado, e ameaçariam a própria saúde de Tiomno. Como decidiu morar no Rio de Janeiro, Tiomno recebeu novo convite de seus antigos discípulos e admiradores Swieca, Videira, Zagury e Erasmo para trabalhar na PUC/RJ. Desse modo, passou a integrar o corpo docente dessa universidade católica, a partir de 1973. É oportuno destacar que, como ele estava proibido pelos militares golpistas de atuar em qualquer instituição de ensino, privada ou pública, houve interferência do papa Paulo VI para que, dessa vez, fosse aceito nessa Universidade.[30]

Contudo, a hostilidade que aqueles militares exerciam contra os professores cassados pelo AI-5, fez com que Tiomno tivesse uma depressão nervosa, que o obrigou a fazer um tratamento terapêutico-analítico, em 1974-1975. Sabedor disso, seu velho amigo Wheeler, por intermédio do matemático norte-americano Bill Faris, que se encontrava no Departamento de Matemática da PUC/RJ, envioulhe uma carta convidando-o para trabalhar na Universidade do Texas, em Austin, para onde Wheeler fora, em 1976, depois de se aposentar na Universidade de Princeton. Em 26 de janeiro de 1978, Tiomno escreve para Wheeler lamentando não poder aceitar o convite pelas razões referidas acima, mas sinaliza a possibilidade de ir para o Texas, no ano seguinte.[31] A abertura política patrocinada pelos generais-presidente Ernesto Geisel e João Batista Figueiredo e a intensificação da luta democrática, que levaram à promulgação da lei da anistia, em 1979, propiciaram a volta de Tiomno para o CBPF, a partir de 1980.

No CBPF, Tiomno retomou seu antigo interesse pela Teoria da Relatividade Especial e, com o físico brasileiro Waldyr A. Rodrigues Junior, preparou um artigo analisando propostas, formuladas por D. G. Torr e P. Kolen, de experimentos que visavam discernir a Teoria da Relatividade Especial de outras teorias, e o submeteu à Foundations of Physics, em 1982. Tiomno e Rodrigues sustentaram que os autores criticados não atingiam seus objetivos, porque 
a análise dos experimentos revelava que eles não eram capazes de estabelecer a distinção pretendida entre a Teoria da Relatividade e outras teorias físicas. O tema era caro a Tiomno, que vinha se dedicando a ele desde 1980. Ao receber a rejeição desse trabalho por parte do editor dessa revista, o físico holandês Alwyn van der Merwe, Tiomno recorreu a Wheeler, em 22 de junho de 1983, para uma opinião sobre essa rejeição. Tiomno explicou que a dificuldade era derivada do fato de que seu artigo revisava, de modo crítico, propostas de experimentos visando contrastar a Teoria da Relatividade com outras teorias, e um dos físicos criticados teria sido escolhido pelo editor da revista para ser árbitro de Tiomno e Rodrigues. Em 13 de setembro de 1983, Wheeler escreveu uma carta a van der Merwe, falando do talento e da honestidade científica de Tiomno e, concluiu que, ... - "unless you know of some truly supervening objection - could pry it loose and have it published". O artigo foi finalmente publicado, em 1985, no volume 15, p. 945.

\section{Wheeler e o Reconhecimento da Contribuição de Tiomno ao Estudo das Interações Fracas}

Vinte anos atrás, Wheeler mobilizou-se ativamente em defesa do reconhecimento, no âmbito da História da Física, das contribuições de Jayme Tiomno. Dessa vez, tratava-se da contribuição de Tiomno para o entendimento das interações fracas. Quando se começou a organizar uma conferência em Racine, Wisconsin, Estados Unidos, sobre os "50 Years of Weak Interactions", que se realizaria de 29 de maio a $1^{\circ}$ de junho de 1984, Wheeler, pressentindo o risco, e impossibilitado de comparecer a essa conferência, escreveu, em 7 de fevereiro de 1984, uma carta para Tiomno, que se encontrava na Universitá degli Studi di Padova, na Itália, apelando, de modo quase imperativo, para que preparasse um artigo sobre a contribuição que dera a esse tema e o apresentasse no evento. A transcrição das palavras de Wheeler pode fornecer uma imagem da dimensão humana e social presente na questão do reconhecimento das prioridades na ciência:

"Terrible, terrible! I have two addresses for you and therefore I don't have any address. But this is too important not to try both: You really must go to the Racine Conference and give a paper under the title 'Early History of the Universal Weak Interaction', or something of the kind. There is a Vietnamese proverb that says, 'He who is absent is wrong'. You will be right and you must be present! I can't. Please cable or telephone D. Cline. You remember Napoleon's words, 'Every hour of lost time is a potential source of disaster".

A intervenção de Wheeler foi providencial, porque Tiomno aceitou o apelo e apresentou o trabalho referido anteriormente: The Early Period of the Universal Fermi Interactions. O trabalho de Tiomno continuou a ser discutido, após a Conferência, em cartas que ele trocou (6 de junho e 14 de setembro de 1984) com Marshak (27 de julho e 28 de setembro de 1984).
Sobre essas cartas, é importante fazer um comentário adicional. Em 1955, Tiomno publicou um artigo no Nuovo Cimento 1, p. 226, intitulado Mass Reversal and the Universal Interaction, no qual chegou à conclusão de que a hipótese da "mass reversal invariance" $\left(\psi \rightarrow \gamma_{5} \psi, m \rightarrow\right.$ $-m$ ), que já havia considerado em sua tese de doutoramento, de 1950, levaria a duas classes de interação de Fermi: S+P-T (Scalar mais Pseudoescalar menos Tensor) ou V-A (Vector menos Axial), com conservação de paridade. Tiomno escolheu a alternativa S+P-T. Ainda hoje, ao relembrar aquela opção, ele afirma: foi "um grande azar", porque me esqueci completamente do trabalho de Elisa".[32] O "trabalho de Elisa" é a experiência com emulsões nucleares realizada pelas físicas brasileiras Elisa Frota-Pessoa e Neusa Margem (hoje, Amato), em 1950 (Anais da Academia Brasileira de Ciências 22, p. 371), que havia mostrado que a desintegração do méson $\pi$ em elétron era, pelo menos, cem vezes menos freqüente que a em méson $\mu$. Isso era incompatível com a presença do pseudoescalar $\mathrm{P}$ na corrente fraca com $\mathrm{S}+\mathrm{P}-\mathrm{T}$. No entanto, o fato de estar no Brasil fora do fluxo de idéias e informações também contribuiu para a decisão de Tiomno, porque foi uma análise cuidadosa de resultados experimentais, disponíveis nos Estados Unidos antes mesmo da publicação, que levou Marshak e Sudarshan, a escolher a alternativa V-A, e foi por eles apresentada na Conference-Venice, ocorrida em Pádua, entre 22 e 28 de setembro de 1957, sem fazer referência ao trabalho de Tiomno. Contudo, no artigo que eles apresentaram na Racine Conference, há uma referência de que o trabalho de Tiomno, de 1955, é um precursor da interação fraca universal V-A. Essa referência é enfatizada na carta de 28 de setembro de 1984, que Marshak escreveu a Tiomno, com a qual enviou-lhe a versão final daquele artigo, conforme o parágrafo final da carta: "In any case, I hope the enclosed version does justice to your contributions to the universal $\mathrm{V}$-A program. It is clear to me that you were aware of the gama5 $\left(\gamma_{5}\right)$ transformation before the rest of us (e.g. your thesis) and that if you had not been so isolated in Brazil, you would have made the same comprehensive analysis of the experimental situation as George (Sudarshan) and I did, and probably arrived at the same V-A conclusion".

O reconhecimento público da contribuição de Tiomno à universalidade da interação fraca fez com que seu grande amigo Wheeler o indicasse para receber o PNF de 1987 , em carta que escreveu para o físico sueco Stig Gunnar Lundqvist, em 6 de fevereiro de 1987. Este havia recebido do físico chinês San Fuan Tuan a proposição ao Comitê Nobel que aquele prêmio fosse atribuído a Sudarshan, Marshak e à física chinesa Madame Chien-Shiung $\mathrm{Wu}$, por haverem contribuído, respectivamente, teórica e experimentalmente, àquela universalidade. Wheeler concordou integralmente com a recomendação, mas com uma adição essencial: a inclusão de Jayme Tiomno. Essa inclusão nessa premiação devia-se, segundo Wheeler, ao fato de ele haver tornado inescapavelmente claro: "The magnitude of the coupling is the same in the beta decay, in mu-meson decay and in the charge-exchange interaction of a mu-meson with a nucleon". No final dessa carta, Wheeler enfatiza que a premiação dos quatro físicos, ... "would doubly emphasize 
to every thinking person the wonderful binding power of science, linking in this way North America (Marshak), South America (Tiomno), India (Sudarshan) and China (Madame Wu)". Os destaques entre parênteses são nossos. Registrese que o PNF de 1987 foi atribuído aos físicos, o alemão Johannes Georg Bednorz e o suíço Karl Alex Muller, pela descoberta das cerâmicas supercondutoras, em 1986.

\section{Everything Is Information}

$\mathrm{Na}$ seqüência deste artigo, vamos examinar a última fase da pesquisa de Wheeler - Everything Is Information que se relaciona, basicamente, ao problema da medida em Mecânica Quântica. Este problema tem suscitado, desde os debates entre Einstein e Bohr na década de 1930, um prolongado debate entre os físicos ao longo do século XX. Este tem sido o tema central que motivou a nova fase de pesquisa de Wheeler, Everything Is Information. Devido à importância do tema para essa nova etapa de seu trabalho científico, Wheeler, com a colaboração do físico Wojciech Hubert Zurek, editou o livro intitulado Quantum Theory and Measurement, publicado pela Princeton University Press, em 1983, e que reúne uma série de artigos que têm a medida quântica como a questão central. O interesse de Wheeler na controvérsia sobre os fundamentos da teoria quântica, a partir de meados da década de 1970, foi também um reflexo de mudanças mais gerais no ambiente intelectual da Física, pois, como argumentado por um dos autores deste artigo [OFJ], naquela época, esses temas deixaram de ser considerados objeto de uma controvérsia filosófica, e por isso externa à física, para serem incorporados à agenda da física, teórica e experimental, como uma controvérsia científica.[33]

Nessa nova fase de pesquisas, Wheeler propôs, em 1978, uma "experiência de pensamento", denominada por ele de "experimento de escolha-demorada" ("delayed-choice experiment"), com a qual ele discute o problema da medida quântica. Como um dos objetivos deste artigo é o de apresentar somente as idéias propostas por Wheeler para o entendimento da física, examinaremos apenas a essência desse "experimento" (o leitor interessado em seus detalhes poderá vê-los no citado livro Geons, Black Hole and Quantum Foam). Vejamos qual é ela. Segundo a mecânica quântica ortodoxa, traduzida pela equação de Schrödinger e pela interpretação de Copenhague, a probabilidade representa um papel central. Assim, no átomo de hidrogênio, por exemplo, $\Psi$ apenas nos diz onde o elétron pode estar, e não onde ele está. Contudo, somente uma adequada experiência pode, de fato, localizar (medir) o elétron em um particular lugar naquele átomo. Portanto, para Wheeler, não é $\Psi$ a essência da mecânica quântica, e sim a medida, que decorre de um "experimento de escolha-demorada". Desse modo, Wheeler conclui que "é o experimento que provê a real informação (grifo nosso)".

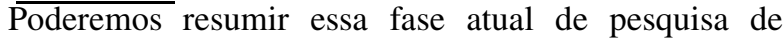
Wheeler, usando suas próprias palavras apresentadas em seu livro Geons:

Medida, o ato de tornar potencialidade em atualidade, é um ato de escolha, escolha entre possiveis resultados. Depois da medida, não há caminhos a serem tomados. Antes da medida, todos os caminhos são possíveis - podemos mesmo dizer que todos os caminhos são considerados de uma única vez. Por fim, Wheeler apresenta sua grande síntese: As leis da física nos dizem somente o que pode acontecer. A medida real nos diz o que está acontecendo (ou o que aconteceu). _A informação é o cerne da física (grifo $\overline{\text { nosso }}$.

Ao concluirmos este artigo, cremos ser oportuno destacar outros aspectos interessantes da vida científica de Wheeler. Em 1937 (Physical Review 52, p. 107), ao estudar a estrutura nuclear, ele considerou uma maneira formal de relacionar cada um dos muitos estados iniciais de um componente do núcleo (próton ou nêutron) com os vários estados finais, sendo todas essas possibilidades englobadas no que ele denominou de matriz de espalhamento. Posteriormente, essa matriz ficou mundialmente conhecida como a famosa matriz $S$, em decorrência dos trabalhos independentes realizados, em 1943, por Heisenberg e pelo físico suíço Ernst Carl Gerlach Stückelberg, ao descreverem a interação de um sistema físico por intermédio dessa matriz, que representa a amplitude de transição entre os estados inicial e final do sistema físico em questão. Uma outra importante contribuição dada por Wheeler para o entendimento da Física foi a que ele desenvolveu com o físico norte-americano Bryce DeWitt. Trata-se da hoje famosa equação de Wheeler-DeWitt (EWD), que adapta a equação de Schrödinger ao espaço curvo da Relatividade Geral. Observe-se que a EWD foi proposta por DeWitt, em 1967 (Physical Review 160, p. 1113), seguindo uma idéia apresentada por Wheeler na década de 1950. Desse modo, segundo essa EWD, se fosse conhecida a forma atual da função de onda do universo ou função de onda de Wheeler-DeWitt (W), ela então permitiria nos dizer qual a probabilidade de o universo visível possuir certas características em larga escala. Em vista disso, a busca de $\mathrm{W}$ se constitui no principal e atual tema da cosmologia quântica.[34]

No arremate deste artigo, é oportuno dizer que Wheeler, assim como grandes físicos norte-americanos e estrangeiros trabalhando na América do Norte, contribuiu para o esforço de guerra dos Estados Unidos, participando do Manhattan Project [construção da bomba atômica (1943-1945)] e, também, do projeto de construção da bomba de hidrogênio (1949-1950). Em sua autobiografia também encontraremos importante informação tanto sobre o seu posicionamento político quanto sobre seu posicionamento em face da corrida armamentista, temas que não comentamos neste artigo.

Por fim, concluímos este artigo fazendo referência à mais recente relação de Wheeler com a física brasileira. Por ocasião das festas de comemoração de seus 90 anos de idade, ocorrida em julho de 2001, Tiomno teve um convite especial para dela participar. Por muito pouco Tiomno não teria comparecido ao evento. O convite foi extraviado, porque foi enviado para o endereço da Academia Brasileira de Ciências. Sem tempo para solicitar financiamento às agências, Tiomno viabilizou sua presença com o apoio da própria Academia.[35] 


\section{Referências}

[1] J. A. Wheeler and K. Ford - Geons, Black Holes and Quantum Foam - a life in Physics, New York: W. W. Norton and Company, 1998. Para as opiniões sobre Tiomno, ver pp. 174176.

[2] Os Wheeler Papers estão depositados na American Philosophical Society Library, em Filadélfia, Estados Unidos. Toda a documentação aqui citada, salvo informação em contrário, pode ser encontrada nas seguintes pastas: Series I - Box Thot - Univ of CA. Sci.\#1, Folder Tiomno, para a documentação até 1975; e Series II - UT at Austin, Box ThTo, Folder Tiomno, para a documentação ulterior. Um dos autores, [OFJ], expressa seu agradecimento a essa instituição pela concessão da John Slater Fellowship, que lhe permitiu consultar esse arquivo, em junho de 2002.

[3] O artigo intitulado Polyelectrons, no qual Wheeler desenvolveu essa tese, foi publicado nos Annals of the New York Academy 48, p. 219, em 1946, e recebeu um prêmio dessa Academia. A tese referida acima começou a ser desenvolvida por Wheeler, em 1934, assim que concluiu seu doutoramento.

[4] Wheeler logo percebeu que o bi-elétron, com grande massa e confinado em um pequeno volume (muito menor que o volume de um próton), poderia radiar os fótons que mantinham a ligação elétron-pósitron e, portanto, tornar-se altamente instável. Desse modo, ele começou a pensar como poderia substituir os fótons ligantes apenas pela ação à distância entre as duas partículas, tendo em vista uma idéia que já havia sido considerada pelos físicos alemães Karl Schwarzschild, Hugo Martin Tetrode e Adriaan Daniel Fokker, respectivamente, em 1903, 1922 e 1929, ao descobrirem que as equações matemáticas que tratam dessa radiação - as famosas equações de Maxwell - apresentam soluções que envolvem tanto os efeitos "avançados", quanto os efeitos "retardados". No entanto, a adoção dos efeitos "avançados" era um contra-senso, pois significava considerar o efeito antes da causa. Como esses cientistas não haviam encontrado um significado físico para tais efeitos, Wheeler começou, em 1938, a pensar como contornar essa dificuldade. Basicamente, Wheeler e Feynman admitiram que, se o Universo fosse formado de um número limitado de matéria, o futuro poderia afetar o passado. Contudo, com a presença no Universo de um número extremamente grande de objetos contendo carga elétrica, todos envolvidos na absorção e remissão de sinais eletromagnéticos que viajam para a frente e para trás no tempo, estes, que afetariam o passado, se cancelariam, restando apenas os primeiros. Cremos ser oportuno destacar que quando Feynman começou a desenvolver a sua interpretação da Mecânica Quântica, entre 1940 e 1941, ele a discutiu com Wheeler. Nessa interpretação, ele partiu da idéia de que, conhecendo-se o estado de uma partícula num certo instante de tempo, saberemos calcular um outro estado dessa mesma partícula em um outro instante de tempo, se somarmos as contribuições de todos os possíveis caminhos da partícula que o levaram de um estado para um outro. Esses caminhos receberam de Wheeler o nome de "histórias". Registre-se que essa Mecânica Quântica Feynmaniana foi apresentada em 1948, na Reviews of Modern Physics 20, p. 367, e as "histórias Wheelerianas" são hoje conhecidas como diagramas de Feynman.

[5] É interessante registrar que Wheeler, em 1998, voltou a referir-se a essa idéia de ação à distância em carta escrita em 5 de fevereiro desse ano aos físicos, os peruanos Armando Leo Bernui e Germán Ignacio Gomero, e os brasileiros Marcelo J. Rebouças e Antonio Fernandes da Fonseca Teixeira. Nessa carta, Wheeler aventou a possibilidade de as "reverberações topológicas" descobertas por esses físicos, em um trabalho prévio enviado a ele, representarem um novo insight físico se fossem descritas em termos daquela ação. Esse trabalho, intitulado Radiation damping in FRW space-times with different topologies, foi publicado na Physical Review D 57, p. 4699, em 1998. Destaque-se que as "reverberações topológicas" (nome cunhado por Wheeler em sua carta e usado por esses físicos em trabalho posterior e mais completo sobre esse mesmo tema, publicado no International Journal of Modern Physics A15, p. 4141, em 2000) decorrem da demonstração apresentada no artigo da $P R$, segundo a qual, em um espaçotempo de Minkowski (plano e usual da Relatividade Especial) com qualquer das dez possíveis topologias não triviais compactas (diferente de $\mathrm{R}^{3}$ ), um sistema irradiante apresenta um efeito de retroalimentação com origem na topologia, e ao invés de perder toda sua energia com o passar do tempo [via amortecimento da radiação ("radiation damping")] apresenta uma curva de energia que não decresce com o tempo devido aos termos não-homogêneos de origem na topologia. Cremos ser oportuno dizer que Bernui e Gomero realizavam no $\mathrm{CBPF}$, respectivamente, pós-doutoramento e doutoramento com Rebouças e, mais ainda, que essa importante descoberta foi realizada em uma instituição de pesquisa brasileira (CBPF), sob a liderança de físicos brasileiros (Rebouças e Teixeira). É também interessante registrar que, ainda nessa carta de Wheeler, ele se refere à descoberta dos eventos elefante (sic!) realizada pelo Grupo de Colaboração Brasil-Japão, sob a liderança, no Brasil, de Lattes, em 1973 (Proceedings of the $13^{\text {th }}$ International Cosmic Ray Conference/IUPAP, Denver-EUA 3;4, p. 2227; 2671) e em 1977 (Nuovo Cimento B42, p. 245). Destaque-se que Rebouças, em resposta àquela carta de Wheeler, escreveu-lhe, em 3 de setembro de 1998, falando sobre os eventos Centauro (e não elefante!), indicando as referências sobre a sua descoberta (que haviam sido solicitadas por Wheeler), assim como destacou que, até aquela data, não havia nenhum modelo teórico satisfatório para explicar esses eventos.

[6] Das várias idéias físicas interessantes trabalhadas por Wheeler nessa primeira fase de sua carreira cabe destacar o modelo da fissão nuclear que formalizou com Bohr. Em 1936, Bohr havia desenvolvido a idéia da "gota líquida" (esta, sugerida antes por Gamow) para explicar as reações nucleares envolvendo a colisão de partículas leves (núcleons: prótons e nêutrons) com o núcleo atômico. Desse modo, a partícula incidente que penetra no núcleo rapidamente reparte sua energia com os núcleons, constituintes nucleares, e perde sua identidade. Assim, Bohr e Wheeler publicaram, em setembro de 1939, dois artigos na Physical Review 56, pgs. 426 e 1056, nos quais usaram aquele modelo para fazer previsões sobre os valores da secção eficaz do núcleo atômico, ao ser bombardeado com nêutrons de diferentes velocidades, como ocorre na fissão nuclear. Note-se que esta havia sido proposta pela física austro-sueca Lise Meitner e por seu sobrinho, o físico austro-britânico Otto Robert Frisch (que cunhou o termo fissão), e independentemente por Bohr, em fevereiro de 1939. Basicamente, Bohr e Wheeler demonstraram que a fissão por nêutrons lentos depende da maior relação entre o 
quadrado do número de prótons e o número total de núcleons. Observe-se que é por essa razão que o ${ }_{92} \mathrm{U}^{235}$ é fissionável por nêutrons lentos e o ${ }_{92} \mathrm{U}^{238}$ não. Este é fissionável por nêutrons rápidos.

[7] O prestígio de Wheeler na física norte-americana do pós II Guerra pode ser avaliado pelo seu papel na organização das conferências de Shelter Island (2-4 de junho de 1947), Pocono (30 de março-2 de abril de 1948) e Oldstone (1114 de abril de 1949), eventos cruciais na projeção da física teórica norte-americana. Ver Silvan S. Schweber - Shelter Island, Pocono and Oldstone - The Emergence of American Quantum Electrodymanics after World War II, OSIRIS, 2nd series, 2, 265-302, 1986. O tema é retomado em S. S. Schweber-QED and the Men Who Made It: Dyson, Schwinger and Tomonaga. Princeton: Princeton University Press, 1994.

[8] Depoimento de Jayme Tiomno e Elisa Frota-Pessoa aos autores e a Sérgio Joffily, Rio de Janeiro, 4 de agosto de 2003.

[9] Val L. Fitch and Jonathan L. Roser - Elementary Particle Physics in the Second Half of the Twentieth Century, in Laurie M. Brown, Abraham Pais \& Sir Brian Pippard (Editors) - Twentieth Century Physics, Volume II, Institute of Physics Publishing and American Institute of Physics, Bristol, Philadelphia \& New York, 1995, p. 776.

[10] Cf. [1], p. 176.

[11] Louis Michel - Coupling Properties of Nucleons, Mesons and Leptons, in J. G. Wilson (Editor) - Progress in Cosmic Ray Physics, New York: North Holland \& Interscience, pp. 125-190, 1952. Ver pp. 174-175.

[12] R. Gourian - Elementos de Física Nuclear (Partículas e Aceleradores), Porto-Portugal: Editoral Inova Limitada, s/d, pp. 110-111. (Registre-se que o livro original foi escrito em 1967)

[13] L. M. Brown and L. Hoddeson (Editors), The Birth of Particle Physics, Cambridge: Cambridge University Press, 1983, p. 395.

[14] Tanto Tiomno quanto Marshak participaram da International Conference on 50 Years of Weak Interactions, realizada entre 29 de maio e $1^{\circ}$ de junho de 1984, em Racine, Wisconsin, EUA. Infelizmente, as atas dessa conferência não foram publicadas. É razoável supor que essa não publicação tenha contribuído para limitar o crédito ao trabalho de Tiomno. Como corroboração dessa suposição, observamos que Abraham Pais - no seu Inward Bound - cita apenas o trabalho de Marshak \& Sudarshan, mas sem a referência de sua publicação. Ver A. Pais - Inward Bound - Of Matter and Forces in the Physical World. New York: Oxford University Press, 1986, p. 547, n.163. Os textos finalmente foram publicados, em 1994, em Alfred K. Mann \& David Cline (eds) - Discovery of Weak Neutral Currents: The Weak Interaction before and after, New York: AIP Press, 1994. O trabalho de Tiomno foi intitulado "The Early Period of the Universal Fermi Interaction" (p.99), e o trabalho apresentado por Marshak, de autoria de E. C. G. Sudarshan \& R. E. Marshak, teve como título "Origin of the Universal V-A Theory" (p.110). Este volume reúne textos de conferência de mesmo título realizada em Santa Mônica, Califórnia, 1993. Somos gratos a Antonio Nassar e David Cline pelo auxílio na recuperação dessa informação.

[15] Em carta a Tiomno, datada de 27 de julho de 1984, Marshak admitiu o equívoco, "your citation of my reference to the
'Puppi triangle' in my article in the Brown-Hoddeson book is understandably scornful", e explica que ele tinha estado bastante isolado do "mainstream" da Física na década anterior, quando ocupara a presidência do City College, e que chamado a fazer aquela palestra usou o "then current term 'Puppi triangle' without further checking".

[16] Robert E. Marshak - Conceptual Foundations of Modern Particle Physics, World Scientific, Singapore, 1993, p. 18.

[17] Wheeler and Fox, op. cit., p. 176.

[18] Em maio de 1952, Wheeler discutiu com o físico canadense Allen Shenstone, chefe do Departamento de Física da Universidade de Princeton, a possibilidade de ensinar a Teoria da Relatividade, no ano de 1953. Como ele não sabia bem essa teoria, e seguindo o apotegma "A melhor maneira de aprender, depois de ensinar, é escrever", começou a preparar as Notas de Aula de seu futuro curso sobre a Relatividade. É oportuno dizer que essas notas foram cada vez mais aumentadas e se transformaram no hoje famoso livro Gravitation, editado em 1973 pela W. H. Freeman and Company, escrito em colaboração com os físicos norte-americanos Charles W. Misner e Kip S. Thorne. Destaque-se que, antes, em 1963, Wheeler já tratara da Teoria da Relatividade ao escrever um livro sobre a Teoria da Relatividade Restrita, em parceria com o físico norte-americano Edwin F. Taylor, intitulado Spacetime Physics e também editado pela Freeman.

[19] Vejamos como Wheeler desenvolveu tais idéias. Quando esteve na Universidade de Leiden, entre janeiro e setembro de 1956, fazendo mais um pós-doutoramento, Wheeler teve a oportunidade de pensar naquela idéia com os físicos, os norte-americanos Misner, Joseph Weber e Peter Putnam, e o italiano Tullio Regge. Com Misner, Wheeler publicou um longo trabalho, nos Annals of Physics-New York 2, p. 525, em 1957, no qual discutiram a gravitação, o eletromagnetismo, a carga não-quantizada e a massa como propriedades do espaço vazio curvado. Nesse artigo, eles mostraram como extrair os campos elétrico e magnético da curvatura do espaço-tempo. Nesse artigo é ainda apresentada a idéia dos "wormholes", que são "buracos" no espaço múltiplo conectado, pelos quais as linhas do campo elétrico (este extraído da curvatura do espaço-tempo, conforme vimos) aparecem e desaparecem. Ora, como na Eletrodinâmica as linhas de forças do campo elétrico vão de uma carga positiva para uma negativa, essa idéia do "wormhole" justificava a afirmativa da idéia que expusera no artigo referido antes sobre geon, isto é: "carga sem carga". Agora, vejamos como Wheeler desenvolveu a idéia da "quantum foam". Ainda em 1957, Wheeler fez um outro trabalho, publicado nos Annals of Physics-New York 2, p. 604, no qual tratou do aspecto quântico da Geometrodinâmica. Nesse trabalho, ele voltou à idéia discutida no trabalho anterior que escrevera com Misner sobre o "wormhole", ou seja, a de que a geometria do espaço tridimensional experimenta uma mudança com a passagem do tempo, e se propaga de um lugar para o outro. Desse modo, o campo gravitacional se propaga de um lugar para o outro, "aparecendo" e "desaparecendo" nos "wormholes". Assim, ao aplicar a Teoria Quântica à Gravitação, ele percebeu que a visão da Gravidade Quântica é uma visão turbulenta do espaço, do tempo e do próprio espaço-tempo. Contudo, essa turbulência só poderia ser observada na chamada escala Planck: comprimento da ordem de $10^{-33} \mathrm{~cm}$, tempo da ordem de $10^{-43} \mathrm{~s}$, energia da ordem de $10^{19} \mathrm{GeV}$ (temperatura da ordem de $10^{32} \mathrm{~K}$ ), e densi- 
dade da ordem de $10^{94} \mathrm{~g} / \mathrm{cm}^{3}$. A essa turbulência (flutuações quânticas do espaço-tempo) Wheeler deu o nome de espuma quântica ("quantum foam”). Esse aspecto do espaço-tempo ("foaminess") decorre do Princípio da Incerteza de Heisenberg e significa dizer que não se pode conhecer, ao mesmo tempo e com infinita precisão, a geometria e o espaço-tempo.

[20] O Teorema acima decorre de trabalhos sobre a Teoria do Buraco Negro que foram escritos por vários físicos, nas décadas de 1950 e 1960. Por exemplo, em 1958, David Finkelstein mostrou a natureza não-singular da singularidade de Schwarzschild. Logo em 1959, C. Fronsdal usou um engenhoso sistema de coordenadas e, com ele, estendeu a métrica de Schwarzschild para descrever completamente a natureza do espaço-tempo até à singularidade de Schwarzschild. O mesmo fizeram Martin David Kruskal e George Szekeres, em trabalhos independentes publicados em 1960. Aliás, é interessante destacar o seguinte fato curioso relativo a esse trabalho de Kruskal. Por volta de 1957, Kruskal discutiu com Wheeler a idéia de contornar a dificuldade matemática no tratamento do espaço-tempo na região em torno da singularidade de Schwarzschild, região mais tarde conhecida como horizonte de eventos de um buraco negro. Como em 1959 Kruskal ainda não havia publicado essa idéia, Wheeler resolveu então apresentá-la em um artigo, e enviou-o para a Physical Review, no nome de M. D. Kruskal. Quando este se encontrava na Alemanha, recebeu com surpresa os "galley proofs" para fazer a revisão. Ao descobrir que fora Wheeler quem o enviara, propôs que o artigo fosse assinado pelos dois. Wheeler não aceitou, e o artigo foi publicado no volume 119 (p. 1743), daquela revista, em 1960.

[21] O então diretor da Faculdade de Filosofia, Ciências e Letras da USP, Prof. Mário Guimarães Ferri, ameaçava-o com o corte de salário e com a possibilidade de demissão sob o pretexto de abandono do serviço. Estas tentativas foram impedidas com a impetração de um mandado de segurança. Ver, Alberto da Rocha Barros (advogado) - Em prol da ciência brasileira - a cátedra de Mário Schenberg, São Paulo, 1965, 50pp.

[22] Cf. nota 8.

[23] Este apoio está largamente documentado na literatura histórica sobre o período. Ver, por exemplo, Elio Gaspari - A Ditadura Envergonhada, São Paulo: Companhia das Letras, 2002, pp. 45-125.

[24] Na mesma pasta dos "Wheeler papers" [Series I - Box Thot - Univ of CA. Sci.\# 1, Folder Tiomno], ao lado de vários recortes de notícias da imprensa americana sobre os acontecimentos políticos no Brasil, encontra-se documentação relativa à criação de um "American Committee for Information on Brazil", criado em New York, em fevereiro de 1970, contando com a participação de religiosos e de artistas [R. D. Abernathy, J. Bennett, T. Cornell], de parlamentares [B. S. Rosenthal] e de acadêmicos [R. Morse, S. J. Stein, T. Skidmore, I. F. Stone].
[25] Sobre a participação de Tiomno na criação do ICTP, ver Alexis De Greiff - The tale of two peripheries: The creation of the International Centre for Theoretical Physics in Trieste. Historical Studies in the Physical and Biological Sciences, 33(1), 33-59, 2002.

[26] Cf. nota 8.

[27] Wheeler papers, Series I, Box Ni-Ph, Folder Nussenzveig.

[28] Cf. nota 8. É oportuno registrar que dois desses trabalhos realizados, em 1972, com M. Davis e Ruffini (Physical Review D 12, p. 2932) e com Ruffini e C. V. Vishveshwara (Nuovo Cimento Letters 3 , p. 211) sobre a radiação gravitacional de corpos caindo nas proximidades de um buraco negro, foram destacados por Martin Rees, Ruffini e Wheeler no livro intitulado Black Holes, Gravitational Waves and Cosmology: An Introduction to Current Research, editado por Gordon and Breach Science Publishers, New York, 1974.

[29] "We are leaving tomorrow looking forward to a vague possibility of remaining in Rio. We were much pleased with our stay in Princeton. I have profited much from it and started a new phase of my career. Even the disagreeable dispute with a colleague has had a positive result to stress my friendship with you and to make me recover from the difficulty of writing papers. Here are some of them, which would not exist without your decisive help. Elisa joins me in sending regards to Janette and hoping we shall see you soon, somewhere ... Jayme. PS. I have also sent a set of preprints to Goldberger".

[30] Conforme depoimento de Jayme Tiomno (nota 8), essa teria sido a explicação, não formalizada, para a concretização desse segundo convite.

[31] A relação da depressão nervosa com o contexto político do país está bem evidenciada na resposta de Tiomno a Wheeler: "Coming to the subject of my 'mental blocks', which I always had, about writing letters, I like to tell you that shortly after I came back to Rio, when I already had a job at the Catholic University and was starting a group in Gen. Relativity, I had a nervous breakdown. It had much to do with being abused and deprived of rights (and ideas) and not being able to react promptly and properly in many instances of my life. This became clear during a psycho-analysis treatment (1974-1975). I have been able to do, since, some work, written two papers, and having two other papers to be written".

[32] Cf. nota 8 .

[33] Ver O. Freire Jr., A Story Without An Ending: The Quantum Physics Controversy 1950-1970, Science \& Education, 12 (56), 573-586, 2003.

[34] Sobre esse fascinante tema, o leitor poderá consultar o livro Três Caminhos para a Gravidade Quântica, publicado pela Rocco, em 2002, do físico norte-americano Lee Smolin, um dos principais teóricos dessa Cosmologia.

[35] Cf. nota 8 . 